\title{
Appendix sepsis tracking along the lateral cutaneous nerve of the thigh
}

\author{
D. Gatt*
}

Ealing Hospital, Uxbridge Road, Southall, Middlesex, UK

\begin{abstract}
Summary: A patient presented with tracking of pus from a chronic appendiceal abscess along the lateral cutaneous nerve of the thigh. In the absence of such a tunnel, which is only present in a small proportion of individuals, the infection would have resulted in a subinguinal psoas abscess. Although pus may conceivably extend in any direction, the reason for this unusual complication remains poorly understood.
\end{abstract}

\section{Introduction}

Acute appendicitis is undoubtedly one of the commonest surgical emergencies in the Western world, yet unusual complications continue to occur. The case reported involves the tracking of a chronic appendix abscess along the lateral cutaneous nerve of the thigh via its canal at the lateral end of the inguinal ligament. This sequence of events does not appear to have been reported previously.

\section{Case report}

A 76 year old man presented with a 2 week history of paraesthesiae along the outer aspect of the right thigh, followed by the development of a painful $8 \mathrm{~cm}$ diameter swelling over the right anterior superior iliac spine. He had been anorexic and had lost weight gradually for several months.

Fifteen months earlier a suspicion of a mass in the right iliac fossa prompted investigation by means of a barium enema which showed no intrinsic colonic abnormality but medial displacement of the caecum by a soft tissue mass lateral to it. On intravenous urography the right kidney was shrunken and nonfunctioning. This was known to be secondary to tuberculosis in 1958. Further investigation of the right iliac fossa mass had been withheld because it was no longer palpable 6 weeks later.

On examination he was cachectic and dehydrated but apyrexial. Abdominally there was fullness and tenderness in the right iliac fossa, with an $8 \mathrm{~cm}$ diameter hemispherical tender lump at the level of the right anterior superior iliac spine. This exhibited a

*Correspondence and present address: D. Gatt F.R.C.S. Dept. of Surgery, Royal Postgraduate Medical School, Hammersmith Hospital, Du Cane Road, London W12 0HS, UK. cough impulse and was apparently reducible with immediate refilling on release. Erythema of the overlying skin was minimal. Sensation in the distribution of the lateral cutaneous nerve of the thigh was impaired. He had a leucocytosis and a raised blood urea.

After adequate rehydration, the lump was explored directly via an incision on its surface. As this was deepened an abscess cavity was entered and $250 \mathrm{ml}$ of pus evacuated. The cavity extended to a mass and a larger cavity in the right iliac fossa via a narrow canal. In view of the obscure nature of the pathological process a laparotomy was performed. The caecum was normal but displaced medially by a mass lateral to it. The caecum was mobilized and the mass confirmed to be a very thick walled abscess cavity surrounding an appendicular stump, $30 \mathrm{~mm} \times 7 \mathrm{~mm}$. A further cavity was present at the level of the hepatic flexure and this, though separate, contained $50 \mathrm{ml}$ of similar pus. As the right kidney had been the seat of previous tuberculosis the direct exploration was extended to this level. However, no perinephric pus was present.

On clearing the pus lateral to the caecum it became apparent that floating across both inner and outer cavities was the lateral cutaneous nerve of the thigh. The collar-stud communication between the two cavities was through the canal for the nerve in the lateral part of the inguinal ligament. Appendicectomy was performed and the abscess cavity and the right paracolic gutter were drained separately. His postoperative recovery was uneventful.

Microscopic examination of the appendicular remnant showed an abundance of transmural scarring with only a few scattered polymorphs. The features suggested that there was no acute inflammation at the time but that appendicitis had occurred in the past with possible frequent flare-ups. Coliform organisms

(C) The Fellowship of Postgraduate Medicine, 1985 
were isolated from the pus taken from the various abscess cavities.

\section{Discussion}

Complications of acute appendicitis are numerous and occur mainly in patients with perforation. Abscesses occur in a variety of situations depending on the orginal position of the appendix, but we could find no report of chronic sepsis tracking along the lateral cutaneous nerve of the thigh. Abscess formation from the retrocaecal position may track upwards to the subhepatic region, to the perinephric space (McGahan, 1982) or to the flank (Deodhar et al., 1982); or else downwards into the pelvis, into a pre-existing hernial sac (McKerrow \& Thompson, 1982) or by penetrating the ilio-psoas fascia to present as a psoas abscess.

The lateral cutaneous nerve of the thigh arises from the dorsal divisions of L2, L3 segments of the lumbar plexus and, running beneath the ilio-psoas fascia, reaches the lateral end of the inguinal ligament. At this point, in a minority of cases, instead of passing into the leg deep to the inguinal ligament, the nerve emerges through a tunnel at the lateral end of the inguinal ligament.

Although fascial planes tend to limit spread of infection across them, such occurrence is not uncommon. Psoas abscess complicating appendicitis is a well

\section{References}

DEODHAR, S.D., JAMMIHAL, H.J. \& PATEL, N.P. (1982). Appendicular abscess presenting as a sinus in the right loin. Journal of Postgraduate Medicine, 28(1), 41.

McGAHAN, J.P. (1982). Perinephric abscess secondary to ruptured retrocaecal appendix diagnosed by computerised tomography. Urology, 19, 217. recognized entity, yet this can only result if retrocaecal infection penetrates the underlying psoas or iliacus fascia. Such spread occurred in our case, but the infection tracked preferentially along the lateral cutaneous nerve of the thigh rather than along the iliopsoas. The paraesthesiae along the distribution of the nerve were almost certainly due to increased tension within the nerve tunnel in the inguinal ligament. The presence of a tunnel was probably responsible for this particular mode of spread, as infection tracking along the more common route taken by the nerve (below the inguinal ligament) would have simply resulted in a subinguinal psoas abscess.

The soft tissue mass shown on barium contrast radiography and felt clinically 15 months previously was undoubtedly the chronic appendiceal abscess. The chronic sepsis manifested itself with weight loss and anorexia, but the penetration of the inguinal ligament must have been a very late event, becoming clinically apparent only 2 weeks before presentation. The microscopic features noted in the appendix are compatible with it having been bathed in pus for a long period of time.

\section{Acknowledgements}

I wish to thank Mr Georges Jantet M.B., F.R.C.S. for his permission to report this case.

McKERROW, W.S. \& THOMSON, H.J. (1982). Unusual complication of perforated appendix. British Medical Journal, 284, 1442. 